\title{
First observation of planet-induced X-ray emission: The system HD 179949
}

\author{
S. H. Saar ${ }^{1}$, M. Cuntz ${ }^{2}$, V. L. Kashyap ${ }^{1}$ and J. C. Hall ${ }^{3}$ \\ ${ }^{1}$ Harvard-Smithsonian Center for Astrophysics, \\ Cambridge, MA 02138, USA \\ email: saar@cfa.harvard.edu, kashyap@cfa.harvard.edu \\ ${ }^{2}$ Department of Physics, University of Texas at Arlington, \\ Arlington, TX 76019-0059, USA \\ email: cuntz@uta.edu \\ ${ }^{3}$ Lowell Observatory, Flagstaff, AZ 86001, USA \\ email: jch@lowell.edu
}

\begin{abstract}
We present the first observation of planet-induced stellar X-ray activity, identified for the HD 179949 system, using Chandra / ACIS-S. The HD 179949 system consists of a close-in giant planet orbiting an F9 V star. Previous ground-based observations already showed enhancements in Ca II $\mathrm{K}$ in phase with the planetary orbit. We find an $\sim 30 \%$ increase in the $\mathrm{X}$-ray flux over quiescent levels coincident with the phase of the Ca II enhancements. There is also a trend for the emission to be hotter at increased fluxes, confirmed by modeling, showing the enhancement at $\sim 1 \mathrm{keV}$ compared to $\sim 0.4 \mathrm{keV}$ for the background star.
\end{abstract}

Keywords. Planetary systems, stars: activity, stars: coronae, stars: individual (HD 179949), stars: late-type, stars: magnetic fields

\section{Introduction}

Planets have been discovered around a large number of stars, mostly by the cycle Doppler shift of their photospheric lines. Most of these planets have been found around F, G, and K-type main-sequence stars. Moreover, about $20 \%$ of these planets are at an orbital distance of $0.1 \mathrm{AU}$ or less (e.g., Butler et al. 2006), commonly referred to as close-in extrasolar giant planets (CEGPs). An interesting question is whether the CEGPs have any effect on the atmosphere of their parent star. Using observed starplanet systems as a basis, Cuntz et al. (2000) were first to propose that CEPGs can increase chromospheric and coronal activity. This effect was thereafter identified through high-quality data, obtained by Ca II K observations by Shkolnik et al. (2003) for five stars (i.e., HD 179949, HD 209458, $\tau$ Boo, 51 Peg, $v$ And). The observations indicated unambigious star-planet activity enhancement in the HD 179949 system. Subsequent results were given by Shkolnik et al. (2005).

The enhancement was found to be phased with the planet orbital period $\left(P_{\text {orb }} \simeq\right.$ $3.09 \mathrm{~d}$ ), and not the (poorly known) stellar rotation period, $P_{\text {rot }}$, estimated to lie between 7 and $11 \mathrm{~d}$. This clearly implies that the Ca II emission enhancement is caused by some form of star-planet interaction. The peak excess amounts to $\sim 0.7 \%$ in the continuumnormalized $\mathrm{K}$ line core strength (which translates to a $\sim 12 \%$ increase in the [basalsubtracted] chromospheric flux; see Saar et al. 2008), and is shifted in phase by $\Delta \phi_{\text {orb }} \sim$ 0.18 from the planet's inferior conjunction. The presence of only one emission peak per $P_{\text {orb }}$ seems to rule out a tidal interaction that would result in a period of $P_{\text {orb }} / 2$; i.e., one peak per tidal bulge. A variety of models have been proposed to explain the observations, which indicate that planet-induced stellar activity enhancements can be an important 


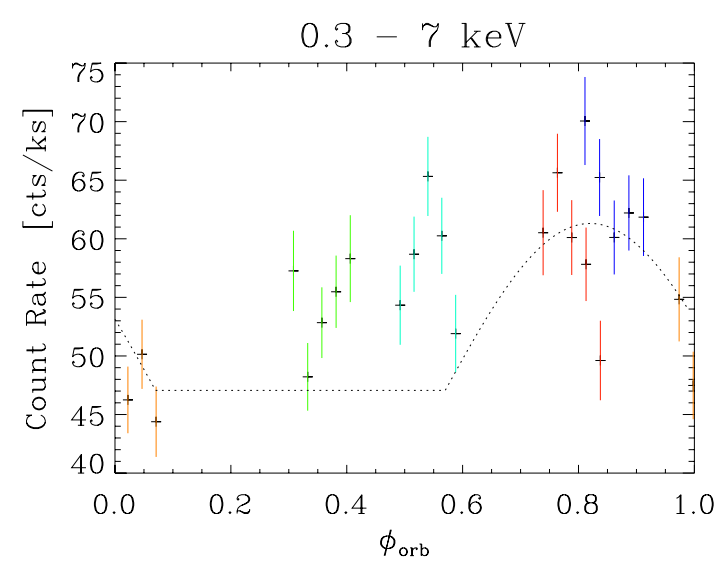

Figure 1. Background subtracted ACIS-S count rate $(0.3-7 \mathrm{keV} ; 6 \mathrm{ksec}$ bins $)$ as a function of the planet orbital phase $\phi_{\text {orb }}$ for various $P_{\text {rot }}$. Observations were taken in $30 \mathrm{ksec}$ segments separated by $1,8,9$, and $10 \times P_{\text {orb }}$ (orange, green, light $\&$ dark blue, respectively) from the first (red). We also show the best fitting Ca II H+K model following Shkolnik et al. (2003) (dotted).

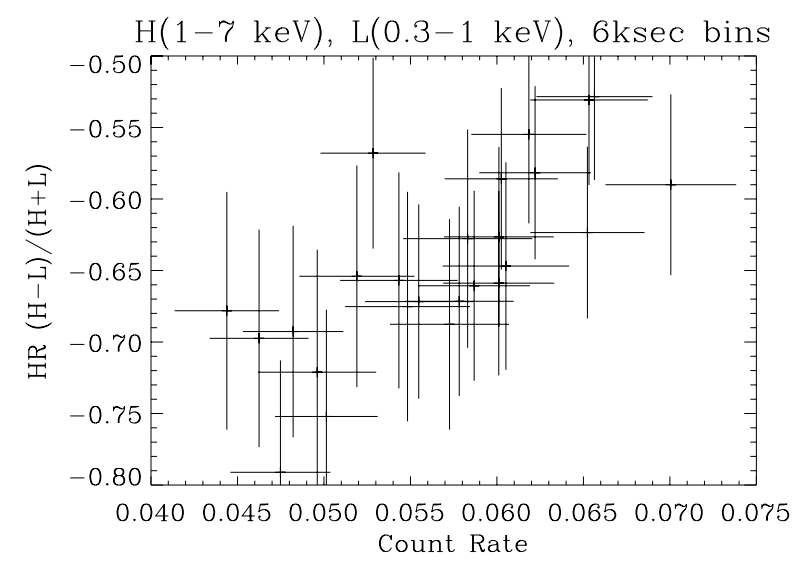

Figure 2. Hardness ratio between high $(1-6 \mathrm{keV})$ and low $(0.3-0.6 \mathrm{keV})$ energies as a function of the total count rate.

probe of (1) the close-in stellar magnetic field structure, (2) stellar wind properties, and/or (3) the planetary magnetosphere (Saar et al. 2004, Grießmeier et al. 2004, Preusse et al. 2005, McIvor et al. 2006, Zarka 2007, Cranmer \& Saar 2008). In the following, we report the first observation of planet-induced X-ray emission, found in the exoplanetary system HD 179949.

\section{Observations and Interpretation}

To reduce complications due to varying stellar activity, the observations were taken at nearly the same stellar rotational phase $\phi_{\text {rot }}$ and within $3 P_{\text {rot }}$, minimizing possible spatial and temporal changes, respectively; see Saar et al. (2008) for details. For $7.5 \mathrm{~d}$ $\leqslant P_{\text {rot }} \leqslant 10 \mathrm{~d}$, the phase span $\Delta \phi_{\text {rot }} \leqslant 0.3$. Plots versus $\phi_{\text {rot }}$ (not given here) show either scatter $\left(P_{\text {rot }}=8-9 \mathrm{~d}\right)$ or sharp changes over multiple orbits difficult to explain with rotational modulation. A Lomb-Scargle periodiogram analysis yields $P=3.289 \mathrm{~d}$ (false 
alarm probability $=2 \times 10^{-11}$ ) with no other $P$ consistent with possible $P_{\text {rot }}$ values; thus a planet-related origin for any variation is preferred.

The data phased to $\phi_{\text {orb }}$ show a minimum around $\phi \sim 0.0-0.1$ and a gradual rise to maximum around $\phi \sim 0.7-0.9$ (Fig. 1). Nearly all the variation is at high energies $(>1 \mathrm{keV})$; additionally, the hardness ratio correlates well with total count rate (Fig. 2 ). The variation at high energies is also not smooth. Thus the "planet effect" produces hot, fluctuating (flare-like?) variability. Contemporaneous $\mathrm{Ca}$ II $\mathrm{H}+\mathrm{K}$ data from the Lowell Observatory Solar-Stellar Spectrograph is consistent (within the large errors) with variations seen in Shkolnik et al. (2005). The best fit to a scaled version of their $\mathrm{H}+\mathrm{K}$ model, however (fixing the minimum to the average flux in $\phi \sim 0.0-0.1$ and the emission peak phase shift $\Delta \phi_{\text {orb }}=0.18$ ), shows significant unmodeled excess flux in the range $\phi \sim 0.4-0.6$ (Fig. 1).

\section{Summary}

We have detected, in the HD 179949 exoplanet system, the X-ray counterpart to the excess $\mathrm{Ca}$ II $\mathrm{H}+\mathrm{K}$ emission previously obtained that is phased to the planet's orbit. The following results are forwarded by the observations:

(a) Peak X-ray enhancement $(0.3-7 \mathrm{keV})$ over the background is $\sim 6$ times that seen in $\mathrm{Ca}$ II $\mathrm{H}+\mathrm{K}$ and shows a similar phase shift from inferior conjunction, which is $\Delta \phi_{\text {orb }} \sim 0.18$.

(b) Thermal plasma models indicate the background has $T \sim 0.4 \mathrm{keV}$, consistent with the corona of a modestly active star. The component responsible for the variability, and associated with the planet, is hotter with $T \sim 1 \mathrm{keV}$ and $\mathrm{EM}_{\text {hot }} / \mathrm{EM}_{\mathrm{cool}} \sim 0.3$.

(c) There is significant additional excess flux around $\phi \sim 0.4-0.6$, which is also hot $(T \sim 1 \mathrm{keV})$, but does not follow the variation seen in $\mathrm{Ca}$ II $\mathrm{H}+\mathrm{K}$. The source of this emission is unclear; it may come from interaction with a second loop (possibly rooted at high stellar latitude) yielding a larger $\Delta \phi$, or it may be emission from the planet's magnetosphere itself (most visible near $\phi=0.5$ ). These different scenarios will be explored in future studies (Saar et al. 2008).

Additional observations and modeling of this phenomenon are needed to obtain further insight into the dominant physical processes.

\section{References}

Butler, R. P., et al. 2006, ApJ, 646, 505

Cranmer, S. R., \& Saar, S. H. 2008, astro-ph/0702530

Cuntz, M., Saar, S. H., \& Musielak, Z. E. 2000, ApJ (Letters), 533, L151

Grießmeier, J. -M., et al. 2004, A\&\&A, 425, 753

McIvor, T., Jardine, M., \& Holzwarth, V. 2006, MNRAS, 367, L1

Preusse, S., Kopp, A., Büchner, J., \& Motschmann, U. 2005, A\&A, 434, 1191

Saar, S. H., Cuntz, M., \& Shkolnik, E. 2004, in: A. K. Dupree \& A. O. Benz (eds.), Stars as Suns: Activity, Evolution and Planets, IAU Symp. 219 (San Francisco: ASP), p. 355

Saar, S. H., Kashyap, V. L., Cuntz, M., Shkolnik, E., \& Hall, J. C. 2008, ApJ, in preparation

Shkolnik, E., Walker, G. A. H., \& Bohlender, D. A. 2003, ApJ, 597, 1092; Erratum 609, 1197 [2004]

Shkolnik, E., Walker, G. A. H., Bohlender, D. A., Gu, P. -G., \& Kürster, M. 2005, ApJ, 622, 1075

Zarka, P. 2007, Planetary and Space Science, 55 (5), 598 